\title{
Serendipitous Spectroscopic Detection of Faint Galaxies at MIR with the IRC Onboard the AKARI During Its In-orbit PV Period
}

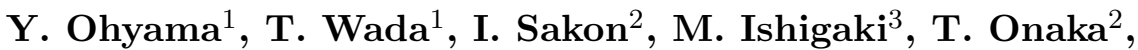 \\ S. Oyabu ${ }^{1}$, and AKARI/IRC team
}

${ }^{1}$ Institute of Space and Astronautical Science, Sagamihara, Kanagwa 229-8510, Japan email: ohyama@ir.isas.jaxa.jp (YO)

${ }^{2}$ Department of Astronomy, The University of Tokyo, Bunkyo-ku, Tokyo 113-0033, Japan

${ }^{3}$ School of Science, Tokyo Institute of Technology, Meguro-ku, Tokyo 152-8551, Japan

\begin{abstract}
The InfraRed Camera (IRC) (Onaka et al. 2004), a wide-field $\left(\sim 10 \times 10 \operatorname{arcmin}^{2}\right)$ NIR-MIR $(2-26 \mu \mathrm{m})$ camera and spectrograph onboard the AKARI (formerly ASTRO-F) space telescope (Murakami et al. 2004), can perform slit-less spectroscopic survey. Many faint galaxies have been serendipitously detected during its in-orbit performance verification period thanks to the power of the slit-less spectroscopy. They are as faint as a few - several mJy in flux, but they can be easily recognized by their prominent spectroscopic features (e.g., PAH). Their redshift $(\lesssim 0.3)$ can be measured through spectral template fitting over the features. We can thus obtain their basic information (redshift, activity type, and luminosity) to investigate nature of galaxies out to much greater distance at MIR than before. With the new IRC/AKARI data, we might be able to address some of the most interesting questions on galaxy evolution at $z \simeq 0.3-2$ (e.g., Genzel \& Cesarsky 2000; Peeters et al. 2004; Pearson 2005): What contributes to the excess found in mid-infrared source count studies with ISO/Spitzer surveys? How does the luminosity function evolve with redshift? How do the spectral features (PAH, etc.) evolve with redshift, and how they correlate with MIR and FIS luminosities with each other? We here demonstrate how MIR galaxy spectra thus obtained are useful for investigation of their basic properties.
\end{abstract}

Keywords. infrared: galaxies

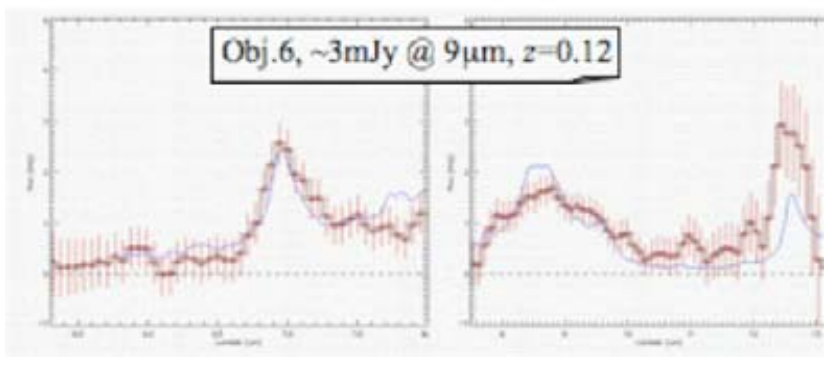

Figure 1. An example spectrum of a galaxy discovered with the IRC slit-less spectroscopy (black steps with red error bars). The galaxy was observed with two grisms, and two spectra are shown side by side. The redshifted $(z \sim 0.1)$ and flux-scaled template spectrum of a nearby galaxy template (NGC 3079) is overlaid (blue).

\section{References}

Murakami, H., et al. 2004, SPIE 5487, 330.

Genzel, R., \& Cesarsky, C. J. 2000, ARAA 38, 761.

Onaka, T., et al. 2004, SPIE 5487, 338.

Pearson, C. 2005, MNRAS 358, 1471.

Peeters, E., Spoon, H. W. W., \& Tielens, A. G. G. M. 2004, ApJ 613, 986. 